\title{
Planetary detection limits taking into account stellar noise
}

\section{Observational strategies to reduce stellar oscillation and granulation effects}

\author{
X. Dumusque ${ }^{1,2}$, S. Udry ${ }^{2}$, C. Lovis ${ }^{2}$, N. C. Santos ${ }^{1,3}$, and M. J. P. F. G. Monteiro ${ }^{1,3}$ \\ 1 Centro de Astrofísica, Universidade do Porto, Rua das Estrelas, 4150-762 Porto, Portugal \\ e-mail: xavier.dumusque@astro.up.pt \\ 2 Observatoire de Genève, Université de Genève, 51 Ch. des Maillettes, 1290 Sauverny, Switzerland \\ 3 Departamento de Física e Astronomia, Faculdade de Ciências da Universidade do Porto, Portugal
}

Received 19 January 2010 / Accepted 15 October 2010

\begin{abstract}
Context. Stellar noise produced by oscillations, granulation phenomena (granulation, mesogranulation, and supergranulation), and activity affects radial velocity measurements. The signature of the corresponding effect in radial velocity is small, around the meterper-second, but already too large for the detection of Earth-mass planets in habitable zones.

Aims. We address the important role played by observational strategies in averaging out the radial velocity signature of stellar noise. We also derive the planetary mass detection limits expected in the presence of stellar noise.

Methods. We start with HARPS asteroseismology measurements for four stars $(\beta \mathrm{Hyi}, \alpha \mathrm{Cen} \mathrm{A}, \mu \mathrm{Ara}$, and $\tau$ Ceti) available in the ESO archive and very precise measurements of $\alpha$ Cen B. This sample covers different spectral types from G2 to K1 and different evolutionary stages, from subgiant to dwarf stars. Since data span between 5 and 8 days, only stellar noise sources with timescales shorter than this time span will be extracted from these observations. Therefore, we are able to study oscillation modes and granulation phenomena without being significantly affected by activity noise present on longer timescales. For those five stars, we generate synthetic radial velocity measurements after fitting the corresponding models of stellar noise in Fourier space. These measurements allow us to study the radial velocity variation due to stellar noise for different observational strategies as well as the corresponding planetary mass detection limits.

Results. Applying three measurements per night of $10 \mathrm{~min}$ exposure each, $2 \mathrm{~h}$ apart, seems to most efficiently average out the stellar noise considered. For quiet K1V stars such as $\alpha$ Cen B, this strategy allows us to detect planets of about three times the mass of Earth with an orbital period of 200 days, corresponding to the habitable zone of the star. Moreover, our simulations suggest that planets smaller than typically $5 M_{\oplus}$ can be detected with HARPS over a wide range of separations around most non-active solar-type dwarfs. Since activity is not yet included in our simulation, these detection limits correspond to a case, which exists, where the host star has few magnetic features and stellar noise is dominated by oscillation modes and granulation phenomena. For our star sample, a trend between spectral type and surface gravity and the level of radial velocity variation is also identified by our simulations.
\end{abstract}

Key words. planetary systems - stars: oscillations - techniques: radial velocities

\section{Introduction}

The majority of planets discovered by Doppler spectroscopy are gaseous giants similar to Jupiter, with masses of a hundred times the mass of the $\operatorname{Earth}^{1}\left(M_{\oplus}\right)$. However, for approximately 5 years, planets from 2 to $10 M_{\oplus}$, have been detected (e.g. Mayor et al. 2009b,a; Udry et al. 2007). This has become possible thanks to the stability and precision of new generation high-resolution spectrographs, and because of dedicated observational strategies allowing us to average out perturbations coming from stellar oscillations (Santos et al. 2004).

Pressure waves ( $\mathrm{p}$-modes) propagate at the surface of solartype stars leading to a dilatation and contraction of external envelopes over timescales of a few minutes (5-15 min for the Sun; Schrijver \& Zwann 2000; Broomhall et al. 2009). The radial-velocity signature of these modes typically varyies between 10 and $400 \mathrm{~cm} \mathrm{~s}^{-1}$, depending on the star type and evolutionary stage (Schrijver \& Zwann 2000). The amplitude and

* Based on observations collected at the La Silla Parana Observatory, ESO (Chile), with the HARPS spectrograph at the 3.6-m telescope.

${ }^{1}$ See The Extrasolar Planets Encyclopaedia, http://exoplanet.eu period of oscillation modes increases with mass along the main sequence. Theory predicts that the frequencies of $\mathrm{p}$-modes increase with the square root of the star mean density and that their amplitudes are proportional to the luminosity over mass ratio (Christensen-Dalsgaard 2004). The most precise spectrograph nowadays, HARPS, can reach a radial-velocity precision of better than the meter-per-second in a short exposure time for bright stars (typically $1 \mathrm{~m} \mathrm{~s}^{-1}$ in $1 \mathrm{~min}$ for a $V=7.5 \mathrm{~K} \mathrm{dwarf}$, Pepe et al. 2005). These acoustic modes are then directly observable and can mask an eventual smallest planet signature.

The different phenomena of granulation ${ }^{2}$ (granulation, mesogranulation, and supergranulation), due to the convective nature of solar-type stars, also affect radial velocity (RV) measurements. These convective phenomena can be found all over the stellar surface, except in active regions where convection is significantly lower (e.g. Dravins 1982; Livingston 1982; Brandt \& Solanki 1990; Gray 1992; Meunier et al. 2010). The amplitudes

\footnotetext{
2 Hereafter, the term granulation phenomena will be used to describe all type of convective motion (granulation, mesogranulation and supergranulation), whereas granulation will just be used for the smaller time scale convective motion.
} 
of granulation phenomena are similar to the ones observed for pressure modes (e.g. Schrijver \& Zwann 2000; Kjeldsen et al. 2005). Granulation corresponds to a small convective pattern with a lifetime shorter than $25 \mathrm{~min}$, and a diameter smaller than 2 Mm (Title et al. 1989; Del Moro 2004). On much larger scales, we can find supergranulation. This phenomenon linked to very large convective patterns from 15 to $40 \mathrm{Mm}$, can have a lifetime up to $33 \mathrm{~h}$ in the Sun (Del Moro et al. 2004). Mesogranulation is a convective phenomenon that can be located between the granulation and supergranulation, in terms of size and lifetime (Harvey 1984; Palle et al. 1995; Schrijver \& Zwann 2000). At the moment, the HARPS-GTO strategy, for the very high precision programs, uses long exposure times $(15 \mathrm{~min})$, to reduce the effects produced by stellar oscillations on RV measurements. However, this does not reduce the low frequency noise coming from granulation phenomena.

On timescales of tenths of days, another type of noise due to the presence of activity-related spots and plages, may perturb precise RV measurements. Spots and plages on the surface of the star break the flux balance between the red-shifted and the blueshifted halves of the star. As the star rotates, a spot, or a plage, moves across the stellar disk and produces an apparent Doppler shift (Saar \& Donahue 1997; Queloz et al. 2001; Huélamo et al. 2008; Lagrange et al. 2010). This modulation can be difficult to differentiate from the Doppler modulation caused by the gravitational pull of a planet. On the Sun, taken as a proxy for $G$ stars, the amplitude of this perturbation can reach $40 \mathrm{~cm} \mathrm{~s}^{-1}$ during high activity phases (Meunier et al. 2010). Spots are cooler than the solar mean surface temperature and plages hotter. Since active regions contain both, the noise induced by spots and plages will usually compensate each other, but not entirely since the surface ratio of spots to plages varies (e.g. Chapman et al. 2001). According to Meunier et al. (2010), the major effect of activity is not due to the break in the flux balance but to the inhibition of convection in active regions. They found that the corresponding noise could vary between $40 \mathrm{~cm} \mathrm{~s}^{-1}$ at minimum activity and $140 \mathrm{~cm} \mathrm{~s}^{-1}$ at maximum.

In this paper, we present simulations exploring new measurement strategies allowing us to average granulation phenomena and oscillation modes at the same time. The detection limits for different strategies are derived, pointing out in particular a strategy that efficiently optimizes detection ability and realistic observational cost. Our simulations also emphasize that for dwarf stars, the level of RV variation, mainly due to stellar noise, is correlated with the spectral type and the surface gravity, $\log g$.

In this study, we focus on understanding the stellar noise acting on timescales shorter than the time span of our data, 8 days. On these timescales, only the influence of oscillation and granulation phenomena can be fully characterized. Longer-term activity signals, typically modulated on timescales of $\sim 30$ days (the usual rotation period of solar-type stars) are not fully accessible. However, noise coming from activity-related phenomena are present in our data. The obtained results will thus only be valid for stars without significant activity phenomena. The effect of plages and spots will be estimated in a forthcoming paper.

\section{Data selection}

To characterize as well as possible the RV noise intrinsic to Sunlike stars, we attempted to find the most precise asteroseismology measurements (continuous follow-up of the star with a high sampling rate). Another requirement was to use only data from one instrument, to avoid any instrumental discrepancies. We thus choose to use only HARPS measurements, since this spectro- graph can reach a RV precision better than $1 \mathrm{~m}$ per second, on the long term (Pepe et al. 2005), and an even better precision, on the level of a few $10 \mathrm{~s}$ of $\mathrm{cm} \mathrm{s}^{-1}$, over one night (Bouchy et al. 2005). Four stars with adequate observations are available in the HARPS ESO archive: $\beta$ Hyi (HD 2151), $\mu$ Ara (HD 160691), $\alpha$ Cen A (HD 128620), and $\tau$ Ceti (HD 10700). The measurements of $\alpha$ Cen B (HD 128621) were provided by F. Pepe (priv. comm.) and do not correspond to continuous measurements. This star was observed for 8 consecutive nights, though only 3 blocks of 20 min (about 27 measurements of $13 \mathrm{~s}$ ) were executed during each night. We later demonstrate that this is sufficient to characterize all type of considered noises. Depending on the star, the total span of the measurements varies from 5 to 8 nights. Table 1 gives more information about the stellar parameters. Our sample contains three main-sequence (MS) stars, one sub-giant, and one star, $\mu$ Ara, which is between the two regimes (Soriano \& Vauclair 2010). This allows us to study a possible correlation between the level of RV variation and spectral type and/or evolution.

Exposure times are always shorter than $100 \mathrm{~s}$. Owing to CCD readout, a dead time of $\sim 30$ s exists between each exposure. This strategy precisely defines the structure of oscillation modes, since they have a typical time scale longer than $5 \mathrm{~min}$. The continuous follow up of the star over 5 to 8 days is essential to characterize convective motions (granulation phenomena), which have time scales up to $\sim 30 \mathrm{~h}$. In the case of $\alpha$ Cen B, the $20 \mathrm{~min}$ of continuous measurements allow us to characterize the granulation, since it has typical time scales shorter than $25 \mathrm{~min}$. The mesogranulation perturbations can be analyzed thanks to the repetition, 3 times a night, of the continuous measurements. Finally, the 8 consecutive nights allow us to address supergranulation. We see in Sect. 3.2 that for the case of $\alpha$ Cen B, all types of noise can be distinguished clearly in the power spectrum, as is the case for the other stars with continuous measurements.

In our sample, $\mu$ Ara is the only star known to have planetary companions. We clearly see in the RVs the effect of the small planet in the system, which we suppress by taking the orbital parameters given in Pepe et al. (2006).

\section{Generating synthetic RV measurements}

In this paper, we study different observational strategies to average out as much as possible the effect of stellar noise on RV measurements. The main goal is to find a strategy capable of detecting very small mass planets in habitable regions. To be able to search for such long-period planets, observational strategies must be efficient over several years. Since the asteroseismology data of the selected stars span only a maximum of 8 days, we have to create longer-term synthetic RV data, containing the same structure and level of noise. To generate these synthetic data, we first calculate the velocity power spectrum density (VPSD) using the periodogram technique. We then fit the obtained spectrum with a noise model and return to the RV space using the property that the periodogram is equivalent to fitting series of sine waves (e.g. Scargle 1982; Zechmeister \& Kürster 2009). The transformation from RVs to the VPSD, as well as the inverse one, does not cause the loss of any information, so the synthetic RV sets should have the same noise structure as the real data we started from. We have however to keep in mind that even if we have synthetic RV data over more days then the real measurements, we do not take into account, in our model, perturbing effects with timescales longer than the real observation time. Therefore, activity noise is not fully included. 
Table 1. Properties of our sample stars.

\begin{tabular}{ccccccccccc}
\hline \hline Star & $M_{V}$ & ${\text { Mass }\left[M_{\odot}\right]^{a}}^{a}$ & $T_{\text {eff }}[\mathrm{K}]^{a}$ & {$[\mathrm{Fe} / \mathrm{H}]^{a}$} & $\log g^{a}$ & $\log \left(R_{\mathrm{HK}}^{\prime}\right)$ & $\mathrm{ST}$ & \# meas. & \# nights/span [days] & $T_{\text {exp }}[\mathrm{s}]$ \\
\hline$\beta$ Hyi & 2.8 & 1.10 & 5872 & -0.11 & 3.95 & - & $\mathrm{G} 21 \mathrm{~V}$ & $2766^{b}$ & $6 / 6$ & 40 to $50^{b}$ \\
$\mu$ Ara & 5.2 & 1.08 & 5780 & 0.30 & 4.27 & -5.20 & $\mathrm{G} 3 \mathrm{IV}-\mathrm{V}$ & $2104^{d}$ & $8 / 8$ & $100^{d}$ \\
$\alpha$ Cen A & 0.0 & 1.09 & 5623 & 0.22 & 4.22 & -5.07 & $\mathrm{G} 2 \mathrm{~V}$ & $4959^{c}$ & $5 / 5$ & 2 to $10^{c}$ \\
$\tau$ Ceti & 3.5 & 0.63 & 5310 & -0.52 & 4.44 & -4.93 & $\mathrm{G} 8 \mathrm{~V}$ & $1962^{e}$ & $5 / 6$ & 23 to $40^{e}$ \\
$\alpha$ Cen B & 1.3 & 0.91 & 5248 & 0.26 & 4.55 & -4.98 & $\mathrm{~K} 1 \mathrm{~V}$ & 783 & $8 / 8$ & 13 \\
\hline
\end{tabular}

Notes. For each star, the dead time due to CCD readout is $31 \mathrm{~s},{ }^{(a)}$ Sousa et al. (2008); ${ }^{(b)}$ Bedding et al. (2007); ${ }^{(c)}$ Bazot et al. (2007); ${ }^{(d)}$ Bouchy et al. (2005); ${ }^{(e)}$ Teixeira et al. (2009). ST is for spectral type and $T_{\exp }$ for exposure time.

\subsection{Velocity power-spectrum density}

We first calculate the velocity power spectrum of the asteroseismology RV measurements. Since the measurements are not evenly spaced in time, we cannot use a standard FFT algorithm. We thus use a weighted Lomb-Scargle periodogram (Lomb 1976; Scargle 1982; Zechmeister \& Kürster 2009) to calculate the power spectrum of the HARPS RV measurements. The minimum frequency of the spectrum is set to $1 / T$, where $T$ is the total span of the observing run and the maximum frequency is set to the Nyquist frequency for the median sampling. To obtain the VPSD, which is independent of the observing window, we multiply the power by the effective length of the observing run, calculated as the inverse of the area under the spectral window (in power, see Kjeldsen et al. 2005).

In the raw VPSD of all the stars, except $\alpha \mathrm{Cen} \mathrm{B}$, we note a substantial excess of power at 3.1, 6.2, and $9.3 \mathrm{mHz}$ (see Fig. 1, top panel). It has been shown that these anomalies are due to a problem in the guiding engine of the telescope, resulting in poor guiding precision (e.g. Teixeira et al. 2009; Bedding et al. 2007; Bazot et al. 2007; Carrier \& Eggenberger 2006). The data for $\alpha$ Cen B, taken in 2009, does not exhibit these peaks because of the corrections made to the guiding software.

To correct this noise effect in our measurements, we use the method developed for $\alpha$ Cen A (Butler et al. 2004), $\beta$ Hyi (Bedding et al. 2007), and $\tau$ Ceti (Teixeira et al. 2009). The idea is to weight poorly bad points produced by the poor guiding. First of all, we compute the raw power spectrum and remove the highest peaks corresponding to oscillations or granulation phenomena. We subtract the related sinusoid component from the $\mathrm{RV}$ data and restart the process of calculating the periodogram and selecting the highest peak until all the power coming from real signals has been removed ${ }^{3}$. The residual velocities obtained should only consist of noise. If this noise is pure photon noise, the residual velocities should respect a Gaussian distribution. For each night, we select all the points deviating from the mean scatter by more than $3 \sigma$ and multiply their statistical weight by the probability of the point belonging to a Gaussian distribution. Finally, we scale all the statistical weights, night by night, to reflect the noise measured at high frequencies. Figure 1 shows, for $\alpha \mathrm{Cen} \mathrm{A}$, the improvement brought about by the cleaning process. After reducing the weight of the poor points, the noise level at high frequencies is reduced by $25 \%$ to $30 \%$ for all stars, except for $\alpha$ Cen B that does not exhibit this guiding noise effect in the raw data. In the case of $\beta \mathrm{Hyi}$, the power spectrum was kindly provided by Tim Bedding; without access to the raw RVs, we could not correct for this guiding noise effect (the peaks at 3.1

\footnotetext{
${ }^{3}$ For $\mu$ Ara, $\alpha$ Cen A and $\alpha$ Cen B, all the peaks below 2.5, 3.5, and $6 \mathrm{mHz}$ are removed, respectively. For $\tau$ Ceti, following Teixeira et al. (2009), we remove all the frequencies below $0.8 \mathrm{mHz}$ and between 3.1 and $5.5 \mathrm{mHz}$.
}

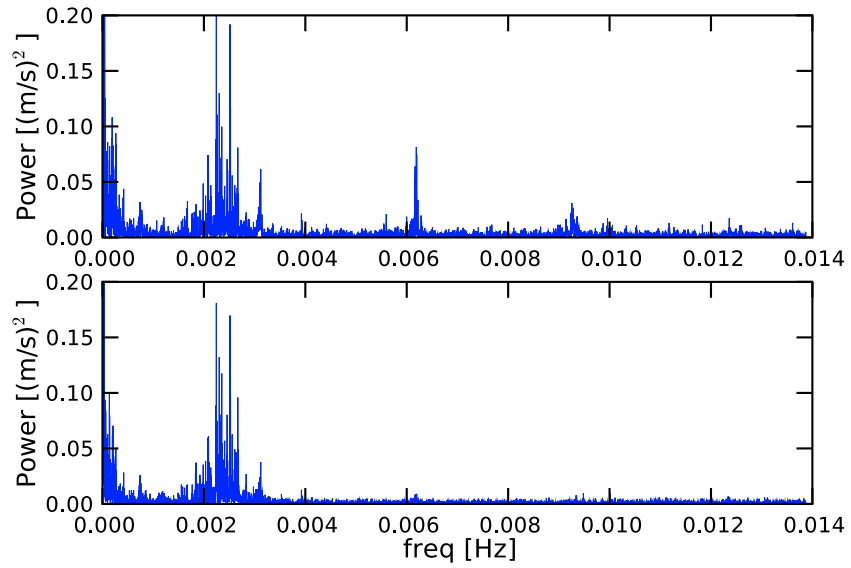

Fig. 1. Cleaning of the guiding noise for $\alpha$ Cen A. Top panel: power spectrum of the RV measurements, using the raw errors to calculate the statistical weights. We can see clearly the peaks at $3.1,6.2$, and $9.3 \mathrm{mHz}$ due to the guiding noise. Bottom panel: same power spectrum but after the cleaning process. The guiding noise peaks are much fainter.

and $6.2 \mathrm{mHz}$ are visible on the $\beta$ Hyi power spectrum in Fig. 2). The corrected VPSD is used in the following study.

For all stars in our sample, we observe an increase in the VPSD towards low frequencies (see Fig. 2) caused by the nature of granulation phenomena. This decrease with frequency follows until reaching the Lorentzian p-modes bump. Finally, after the signature of oscillation modes, we can see that the amplitude of the VPSD remains at the same level because of the photon and instrumental noise.

\subsection{Fitting the velocity power spectrum density}

Once the VPSD is calculated, we need to fit it to obtain a spectrum that is independent of the original data sampling. This will also allow us to separate individually each type of noise, which will give us an idea about the RV contribution of each of them.

The part of the VPSD corresponding to granulation phenomena can be explained by a model in which each source of convective motions is described by an empirical law initially proposed by Harvey (1984) and reviewed by Andersen et al. (1994), and Palle et al. (1995). This model corresponds to an exponentially decaying function with frequency

$P(v)=\frac{A}{1+(B v)^{C}}$,

where $P(v)$ is the VPSD, $A$ the power density of the corresponding convection motion, $B$ its characteristic timescale, and $C$ the slope of the power law. The power remains approximatively constant on timescales longer than $B$ and drops off for shorter 

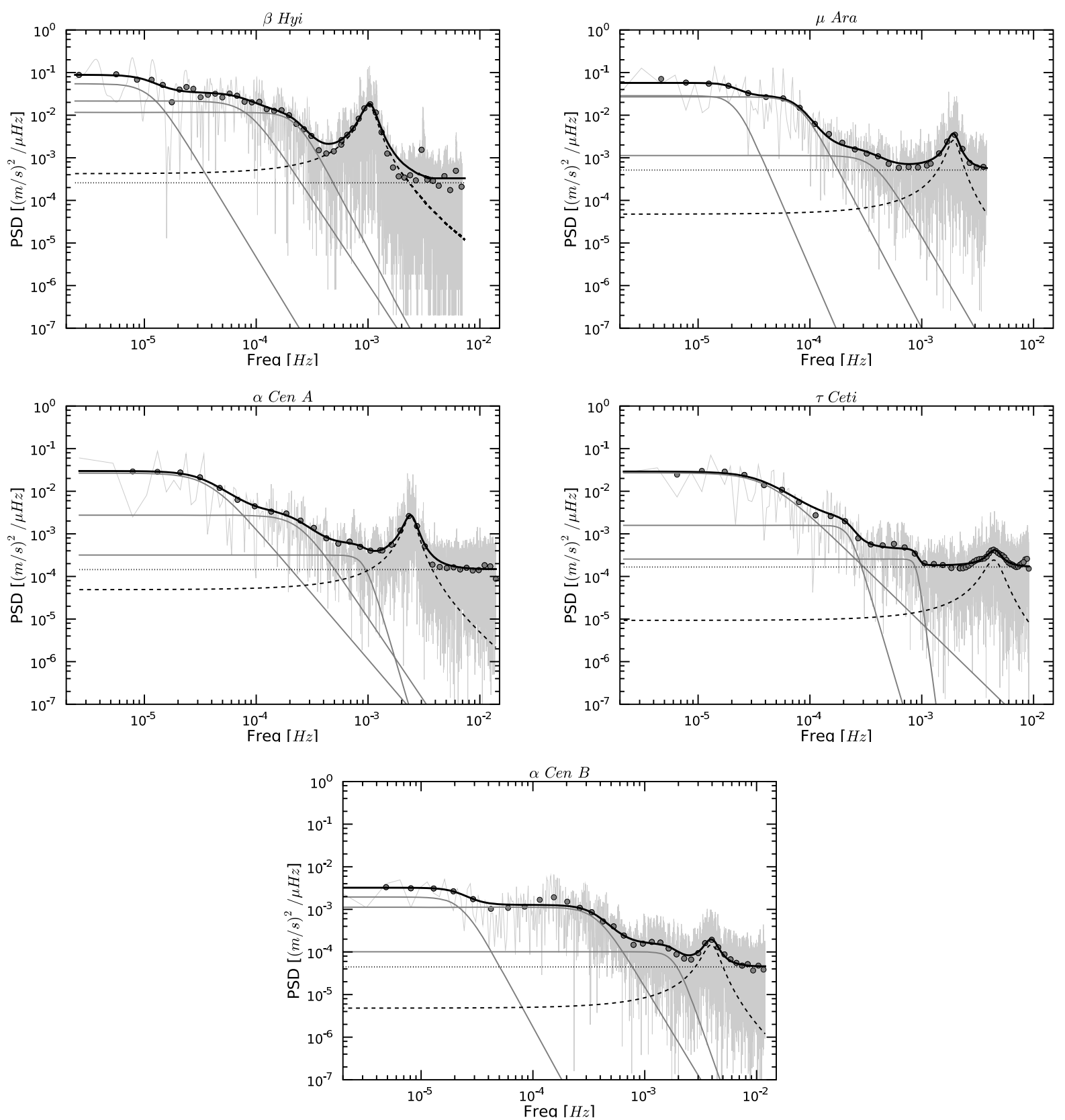

Fig. 2. VPSDs and fits for the 5 stars of our samples. The grey dots corresponds to the binned spectrum using a boxcar algorithm with a changing width according to the frequency. The global fit including granulation phenomena, oscillation modes, photon noise and instrumental noise (black line) is obtained on the binned spectrum. Each contribution to the fit is shown. We can see the 3 types of granulation fitted in light grey lines (supergranulation, mesogranulation and granulation from left to right respectively). The Lorentzian fit to p-modes corresponds to the dashed line and the constant, fitting the photon and instrumental noises, can be see in dotted line.

timescales. The total VPSD due to granulation phenomena is the sum of the VPSD for each phenomenon $(\mathrm{G}=$ granulation, $\mathrm{MG}=$ mesogranulation, and $\mathrm{SG}=$ supergranulaion)

$P_{\mathrm{Tot}}(v)=P_{\mathrm{G}}(v)+P_{\mathrm{MG}}(v)+P_{\mathrm{SG}}(v)$.

The frequency of the oscillation modes (p-modes) can be analytically derived using a perturbation theory and the asymptotic theory (Tassoul 1980), leading to

$v_{n l} \sim \Delta v\left(n+\frac{l}{2}+\alpha\right)$,

where $\Delta v$ is the large separation, $n$ the radial order, and $l$ the angular degree of the mode. We are interested in the total power due to p-modes and not in each mode separately. We thus use the technique proposed by Kjeldsen et al. (2005) (see also Arentoft et al. 2008) to produce a single hump of excess power that is insensitive to the oscillation spectrum having discrete peaks. This is achieved by convolving the observed VPSD with a Gaussian having a full width at half maximum ${ }^{4}$ of $4 \Delta v$. Finally, we fit the

${ }_{4}$ The value of $\Delta v$ for each star of our sample is taken from the literature: $57.2 \mu \mathrm{Hz}$ for $\beta \mathrm{Hyi}$ (Bedding et al. 2007), $106 \mu \mathrm{Hz}$ for $\alpha$ Cen A (Kjeldsen et al. 2005), $90 \mu \mathrm{Hz}$ for $\mu$ Ara (Bazot et al. 2005), $169 \mu \mathrm{Hz}$ for $\tau$ Ceti (Teixeira et al. 2009), and $162 \mu \mathrm{Hz}$ for $\alpha$ Cen B (Kjeldsen et al. 2005) 
convolved curve with a Lorentzian function (e.g. Lefebvre et al. 2008)

$P(v)=A_{\mathrm{L}} \frac{\Gamma^{2}}{\left(v-v_{0}\right)^{2}+\Gamma^{2}}$,

where $A_{\mathrm{L}}$ corresponds to the amplitude of the Lorentzian, $\Gamma$ to the full width at half maximum (FWHM), and $v_{0}$ to the mean of the Lorentzian.

At very high frequency, after the p-modes bump, we can see the contribution of photon and instrumental noises. Assuming that these noises are Gaussian, which is at least the case for photon noise, their contribution corresponds to a constant power over all the spectrum.

To summarize, the entire VPSD can be adjusted by the following function with 13 free parameters

$P(v)=\sum_{i=1}^{3} \frac{A_{i}}{1+\left(B_{i} v\right)^{C_{i}}}+A_{\mathrm{L}} \frac{\Gamma^{2}}{\left(v-v_{0}\right)^{2}+\Gamma^{2}}+$ const.

where $i$ corresponds to each type of granulation phenomena. To perform the VPSD adjustment with 13 free parameters, we bin the spectrum, using a boxcar algorithm ${ }^{5}$, and use an iterative approach. We first adjust the Lorentzian and the constant on the spectrum part corresponding to the oscillation modes, and then, fit the Harvey functions to the low frequency part of the spectrum, taking into account the constant derived before. Finally, we derive a global simultaneous fit of the model including the Harvey functions, the Lorentzian, and the constant using as initial conditions the results of the individual fits. The VPSD and final adjustment for each star can be seen in Fig. 2 and the different parameters, in Table 2.

For the case of $\alpha \mathrm{Cen} \mathrm{B}$, the observation data we have does not correspond to continuous follow-up of the star. To verify that it does not affect high frequency noise, we compare the high frequency spectrum of our data with one night of asteroseismology performed on $\alpha$ Cen B in 2004 (HARPS ESO archive). We find the same p-modes envelope in both cases, which proves that the data we have for $\alpha$ Cen B are as good as the one gathered for the four other stars (see Fig. 2).

The oscillation modes of all the stars of our sample have been previously studied in detail. We thus can compare the mean frequency of the p-modes bump fitted in this paper, with the one found in previous studies (Arentoft et al. 2008; Kjeldsen et al. 2008, and reference therein). According to Fig. 11 in Arentoft et al. (2008), the mean frequency of the p-modes bump are the following: $1 \mathrm{mHz}$ for $\beta \mathrm{Hyi}, 2 \mathrm{mHz}$ for $\mu \mathrm{Ara}, 2.5 \mathrm{mHz}$ for $\alpha$ Cen A, $4.5 \mathrm{mHz}$ for $\tau$ Ceti, and $4 \mathrm{mHz}$ for $\alpha$ Cen B. These frequencies can be compared with our results ( $\operatorname{see} v_{0}$ values in Table 2) and we find very good agreement.

It is more complex to compare the fitted parameters of granulation phenomena with the literature values. To our knowledge, only the Sun has been previously studied in detail (Lefebvre et al. 2008; Palle et al. 1995). In addition, the normalization of the algorithms used by these authors to calculate the VPSD differs from the one adopted here. This implies that different amplitudes are found for the considered granulation phenomena. Some work could be done to normalize the values, but this is beyond the scope of the present paper. We can nevertheless compare the different timescales obtained. In Lefebvre et al. (2008) and Palle et al. (1995), we have access to

\footnotetext{
5 The width of the box changes according to the frequency. The goal is to obtain a binned spectrum which has nearly the same spacing between the points in a logarithmic scale, which makes fit convergence faster.
}

the characteristic time $\tau$, which is by definition equal to $B /(2 \pi)$. Changing the characteristic times from $\tau$ to $B$, Lefebvre et al. (2008) obtain $B_{\mathrm{G}}=22 \mathrm{~min}$, whereas Palle et al. (1995) get $B_{\mathrm{G}}=$ $39 \mathrm{~min}$. In our case, for $\alpha$ Cen A (G2V) and $\mu$ Ara (G3IV-V), which are close to the Sun in terms of spectral class, we find $B_{\mathrm{G}}=18 \mathrm{~min}$ and $44 \mathrm{~min}$, respectively, which are in good agreement. For the cases of meso- and supergranulation, we find some discrepancies in the literature. In Palle et al. (1995), $B_{\mathrm{MG}} \sim 17 \mathrm{~h}$ and $B_{\mathrm{SG}} \sim 175 \mathrm{~h}$. According to more precise and recent results, we can extract roughly from Fig. 2 in Lefebvre et al. (2008) that $B_{\mathrm{SG}} \sim 2 \times 10^{-5} \mathrm{~Hz} \sim 14 \mathrm{~h}$. We note that our results for $\alpha$ Cen A $(\mathrm{G} 2 \mathrm{~V})$ and $\mu$ Ara $(\mathrm{G} 3 \mathrm{IV}-\mathrm{V})$ are close to this second value.

\subsection{Synthetic radial velocity measurements}

Once the VPSD fit is obtained, we have to calculate the RV for each date of an observational calendar. Since the periodogram technique is equivalent to a weighted sine-wave fitting (Scargle 1982; Zechmeister \& Kürster 2009), to pass from the spectrum to RVs we have to calculate for the desired epochs $t_{i}$

$R V\left(t_{i}\right)=\sum_{v} \sqrt{\operatorname{VPSD}(v)}\left(\sin \left(2 \pi v t_{i}+\operatorname{phase}(v)\right)\right)$,

where the frequency $v$ goes from $1 / T$ to the Nyquist frequency in steps of $1 / T$. Assuming that all the components of noise due to stellar perturbations are independent, we choose a uniformly random phase ${ }^{6}$ between 0 and $2 \pi$.

To check whether computation of synthetic RV measurement over long periods does not introduce any noise, which is absent from the asteroseismology measurements, we compute the rms of synthetic RV measurements for different time spans. To be consistent with the observational strategies we use afterwards, we calculate the rms of one measurement per night of $15 \mathrm{~min}$ for 10, 50, 100, and 1000 consecutive nights. For each star, each measurement of $15 \mathrm{~min}$ is divided into several exposures according to the exposure time of the asteroseismology run (see Table 1). The difference between the synthetic RV rms for each time span and the rms of the real RV is smaller than $10 \%$ for all the stars. This corresponds to less than $25 \mathrm{~cm} \mathrm{~s}^{-1}$, which is significantly below the long-term precision level of HARPS (60-80 $\mathrm{cm} \mathrm{s}^{-1}$, HARPS team, priv. comm.). This proves that computation of synthetic measurements over long periods does not introduce any bias. Therefore, these measurements contain only short-term perturbations (oscillations, granulation phenomena, photon and instrumental noise, and a little bit of activity) even if the total time span is much longer.

Table 3 gives the rms of both the real RVs, $\mathrm{rms}_{\mathrm{data}}$, and the synthetic RVs for 100 consecutive nights, $\mathrm{rms}_{\text {syn, tot }}$. We also calculate the rms expected for each noise contribution as well as the high frequency noise derived using the power spectrum. This high frequency noise corresponds to the median level of the power spectrum for frequencies higher than $\mathrm{p}$-modes. We note the good agreement between the rms calculated using the fitted constant and using the power at high frequency, which guarantee a correct estimation of the photon and instrumental noise.

As already pointed out in Mayor et al. (2003), we observe for dwarf stars a trend between the level of RV variation and spectral type. If we look at the main-sequence stars in our sample ( $\alpha$ Cen A (G2V), $\mu$ Ara (G3IV-V), $\tau$ Ceti (G8V), and $\alpha$ Cen B $(\mathrm{K} 1 \mathrm{~V}))$, we note a decrease in the level of RV variation from $\mathrm{G}$

${ }^{6}$ Using the phase calculated by the periodogram is not what we want because it will reproduce the real data, setting the RVs to 0 during the observation gaps. 
Table 2. Parameters for the spectrum fit.

\begin{tabular}{|c|c|c|c|c|c|c|c|c|c|c|c|c|c|}
\hline Star & $A_{\mathrm{SG}}$ & $B_{\mathrm{SG}}[\mathrm{h}]$ & $C_{\mathrm{SG}}$ & $A_{\mathrm{MG}}$ & $B_{\mathrm{MG}}[\mathrm{h}]$ & $C_{\mathrm{MG}}$ & $A_{\mathrm{G}}$ & $B_{\mathrm{G}}[\mathrm{min}]$ & $C_{\mathrm{G}}$ & $A_{\mathrm{L}}$ & $\Gamma[\mathrm{mHz}]$ & $v_{0}[\mathrm{mHz}]$ & Constant \\
\hline$\beta$ Hyi & 0.055 & 24.3 & 4.3 & 0.021 & 3.4 & 4.0 & $11.6 \times 10^{-3}$ & 72.8 & 5.0 & $18.5 \times 10^{-3}$ & 0.17 & 1.0 & $2.6 \times 10^{-4}$ \\
\hline$\mu$ Ara & 0.029 & 13.0 & 6.0 & 0.027 & 3.4 & 5.0 & $1.1 \times 10^{-3}$ & 43.8 & 4.5 & $3.2 \times 10^{-3}$ & 0.26 & 1.9 & $5.1 \times 10^{-4}$ \\
\hline$\alpha$ Cen A & 0.027 & 7.4 & 3.1 & 0.003 & 1.2 & 3.9 & $0.3 \times 10^{-3}$ & 17.9 & 8.9 & $2.6 \times 10^{-3}$ & 0.36 & 2.4 & $1.4 \times 10^{-4}$ \\
\hline$\tau$ Ceti & 0.027 & 6.7 & 2.6 & 0.002 & 1.2 & 8.9 & $0.3 \times 10^{-3}$ & 18.5 & 19.8 & $0.3 \times 10^{-3}$ & 0.75 & 4.5 & $1.7 \times 10^{-4}$ \\
\hline$\alpha$ Cen B & 0.002 & 12.0 & 4.8 & 0.001 & 0.7 & 4.4 & $0.1 \times 10^{-3}$ & 8.9 & 7.5 & $0.2 \times 10^{-3}$ & 0.68 & 3.9 & $0.5 \times 10^{-4}$ \\
\hline
\end{tabular}

Notes. $\mathrm{SG}=$ supergranulation, $\mathrm{MG}=$ mesogranulation, $\mathrm{G}=$ granulation. $A_{\mathrm{SG}}, A_{\mathrm{MG}}, A_{\mathrm{G}}, A_{\mathrm{L}}$, and the constant are expressed in power density, $\frac{(\mathrm{m} / \mathrm{s})^{2}}{\mu \mathrm{Hz}}$.

Table 3. Comparison of the dispersion in real RVs, $\mathrm{rms}_{\mathrm{data}}$, with the synthetic data dispersions for all type of noise, $\mathrm{rms}_{\mathrm{syn}, \text { tot }}$, and for each type of noise individually.

\begin{tabular}{|c|c|c|c|c|c|c|c|c|}
\hline Star & $\begin{array}{l}\mathrm{rms}_{\text {data }} \\
{\left[\mathrm{m} \mathrm{s}^{-1}\right]}\end{array}$ & $\begin{array}{c}\mathrm{rms}_{\mathrm{syn}, \mathrm{tot}} \\
{\left[\mathrm{m} \mathrm{s}^{-1}\right]}\end{array}$ & $\begin{array}{c}\mathrm{rms}_{\mathrm{syn}, \mathrm{SG}} \\
{\left[\mathrm{m} \mathrm{s}^{-1}\right]}\end{array}$ & $\begin{array}{c}\mathrm{rms}_{\text {syn, MG }} \\
{\left[\mathrm{m} \mathrm{s}^{-1}\right]}\end{array}$ & $\begin{array}{l}\mathrm{rms}_{\mathrm{syn}, \mathrm{G}} \\
{\left[\mathrm{m} \mathrm{s}^{-1}\right]}\end{array}$ & $\begin{array}{c}\mathrm{rms}_{\text {syn, } \mathrm{p}-\text { modes }} \\
{\left[\mathrm{m} \mathrm{s}^{-1}\right]}\end{array}$ & $\begin{array}{c}\mathrm{rms}_{\text {syn, const. }} \\
{\left[\mathrm{m} \mathrm{s}^{-1}\right]}\end{array}$ & $\begin{array}{c}\mathrm{rms}_{\text {high frequency }} \\
{\left[\mathrm{m} \mathrm{s}^{-1}\right]}\end{array}$ \\
\hline$\beta$ Hyi & 2.60 & 2.88 & 0.55 & 1.05 & 1.26 & 2.23 & 0.94 & - \\
\hline$\mu$ Ara & 1.97 & 1.90 & 0.56 & 1.05 & 0.46 & 1.11 & 1.00 & 1.08 \\
\hline$\alpha$ Cen A & 2.03 & 1.89 & 0.77 & 0.62 & 0.40 & 1.20 & 0.99 & 1.1 \\
\hline$\tau$ Ceti & 1.47 & 1.42 & 0.85 & 0.43 & 0.34 & 0.52 & 0.87 & 0.96 \\
\hline$\alpha$ Cen B & 0.9 & 0.88 & 0.15 & 0.48 & 0.32 & 0.39 & 0.52 & 0.52 \\
\hline
\end{tabular}

Notes. $\mathrm{SG}=$ supergranulation, $\mathrm{MG}=$ mesogranulation, $\mathrm{G}=$ granulation, const. = instrumental and photon noise. The dispersion in synthetic measurement is calculated during 100 consecutive days, with one measurement per night of $15 \mathrm{~min}$. $\mathrm{rms}_{\text {high frequency }}$ corresponds to the noise calculated in the power spectrum at high frequency, after p-modes.
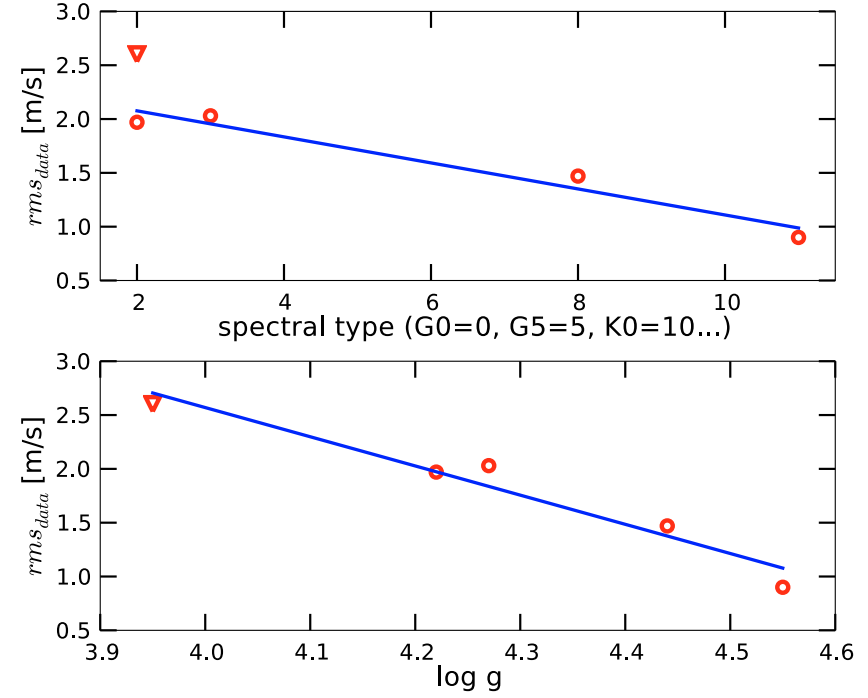

Fig. 3. Top panel: RV variation, $\mathrm{rms}_{\text {data }}$, in function of the spectral type. The fit is done on dwarf stars (circles) without taking into account the sub-giant (triangle). Down panel: RV variation as a function of the surface gravity, $\log g$. The linear fit is performed on all the sample.

to $\mathrm{K}$ dwarfs. Figure 3 (top panel) clearly illustrates this trend. A similar behavior can also be discerned by comparing the RV variation with surface gravity, $\log g$. In this case, all the stars follow the trend, even $\beta$ Hyi (G2IV), which is a subgiant. Thus, the RV variation appears to depend on the spectral type and the evolutionary stage of the star. The expected results on averaging the stellar noise will then strongly depend on the characteristics of the star considered.

Since the fit is the sum of each noise contribution (see Eq. (5)) we can extract each component of noise independently from the VPSD and calculate the corresponding rms. Except for supergranulation, we can see in Table 3 that the noise level of granulation phenomena, as well as oscillations, decreases when we go towards late spectral type stars (the stars are ordered from early spectral type to late spectral type and from evolved star to non-evolved). The calculation of the noise rms induced by supergranulation is very sensitive to the parameters $\mathrm{B}$ and $\mathrm{C}$ as well as the minimum frequency of the spectrum, which explains why we do not see any trend between the noise level of supergranulation and the spectral type. The variation in the noise noise amplitude as a function of the spectral type and evolution is more clearly seen in Table 2 by comparing the values for $A_{\mathrm{SG}}, A_{\mathrm{MG}}$, $A_{\mathrm{G}}$, and $A_{\mathrm{L}}$. In this case, we can see the dependence for supergranulation. Kjeldsen \& Bedding (1995), Christensen-Dalsgaard (2004), and O'Toole et al. (2008) illustrated the dependency of the amplitude of the stellar noise on both the spectral type and evolution of the oscillation modes. To our knowledge, this is the first time that this dependence has been discovered for granulation phenomena. In conclusion, early $\mathrm{K}$ dwarfs have a total noise level lower than $\mathrm{G}$ dwarfs or sub-giants, making them more suitable candidates for the investigation of exoplanets using precise radial-velocity measurements.

All the stars in our sample are very bright, i.e. brighter than magnitude 5.2 (see Table 1), which induce a very small photon noise. For the faintest star $\mu$ Ara, the photon noise estimated for a single exposure of the asteroseismology run is below $34 \mathrm{~cm} \mathrm{~s}^{-1}$. Comparing this value with the one found for the photon and instrumental noise (see $\mathrm{rms}_{\mathrm{syn} \text {, const. }}$ in Table 3), we see that the precision is limited by the instrument. We can separate our sample into two. On the one hand, $\alpha \mathrm{Cen} \mathrm{B}$, which has a very low photon and instrumental noise, and on the other hand, the other stars. Measurements for $\alpha$ Cen B are very recent and much work has been done to reduce the effects of guiding noise, which explains the rms difference between the two samples. In the sample with high values of rms, all the stars seem to have similar instrumental noises, of around $0.9-1 \mathrm{~m} \mathrm{~s}^{-1}$. Several factors may be responsible for the small rms difference. For example, weather conditions play an important role in determining the instrumental noise, mainly guiding noise. If we look at a star with a very good seeing, smaller than the spectrograph fiber diameter 
X. Dumusque et al.: Stellar noise and planetary detection. I.

(1 arcesec), the point spread function of the star will not cover the entire fibre. This introduces a noise when the star changes position in the fiber because of imperfect scrambling. This noise can be averaged for long exposure times, but not for high observational frequencies as requested by asteroseismology.

\subsection{Correcting instrumental noise for long periods}

As shown in the last paragraph, our RV measurements appear to be instrumental noise limited. This noise originate mainly in the telescope guiding system, which is not optimized to observe stars using very short exposure times. For planet surveys, we normally use exposure times of $15 \mathrm{~min}$, which allow a good averaging of the guiding noise and should ensure a smaller instrumental noise. Moreover, estimating the instrumental noise using asteroseismology measurements gives a good approximation of the noise on short timescales, but not on the long timescales required for planet surveys. For long time scales and 15-min exposure time per measurement, the instrumental noise on HARPS is estimated to be between 0.6 and $0.8 \mathrm{~m} \mathrm{~s}^{-1}$ (HARPS team, private communication). To take into account long-term instrumental effects and remove short-term perturbations due to guiding, yet keep the same contribution from oscillations and granulation phenomena, we readjusted the level of the fitted constant to $0.8 \mathrm{~m} \mathrm{~s}^{-1}$ for all the stars and recalculated the synthetic RVs. This value of $0.8 \mathrm{~m} \mathrm{~s}^{-1}$ was assumed to be conservative.

\section{Considered observing strategies}

Using the synthetic RVs for each of the considered stars, our goal is to estimate, for different observational strategies, the variation level of binned RVs induced by stellar noise.

\subsection{Present HARPS-GTO observational strategy}

To be useful in a practical way, simulations have to take into account a realistic access to telescope time. A suitable approach is provided by the scheduling of the high-precision HARPSGTO program. A star such as HD 69830 (see Lovis et al. 2006, 2010 , in preparation for additional points) was monitored using an ideal calendar of one measurement per night on 10 consecutive nights per month over more than 4 years. In the present study, we use this ideal calendar on 4 years and remove 4 month per year, since most of the stars disappear from the sky during this period. In addition, we suppress randomly data for $20 \%$ of the nights, to take into account bad weather or technical problems. This calendar thus represents 256 nights of measurements over a time span of 4 years.

For each star in our sample, we calculate the RV expected for each date of the calendar using an exposure time of $15 \mathrm{~min}$, corresponding to the present HARPS-GTO observational strategy. This strategy will be referred to hereafter as the $1 \mathrm{~N}$ strategy. We then make bins of 2, 5, and 10 consecutive nights and calculate the rms of the binned RVs, $\mathrm{rms}_{\mathrm{RVb}}$, as a function of the binning in days.

The result for each star is illustrated by the heavy solid black lines with circle markers in Fig. 4. For example, in the case of $\alpha$ Cen B, we can see that with a binning in days of 10 , we can reduce the $\mathrm{RV}$ variation, mainly due to stellar noise, to the level of $0.38 \mathrm{~m} \mathrm{~s}^{-1}$, to be compared with $0.94 \mathrm{~m} \mathrm{~s}^{-1}$ in the case without binning.
Table 4. Characteristics of the different observational strategies.

\begin{tabular}{cccc}
\hline \hline $\begin{array}{c}\text { Measure } \\
\text { spacing [h] }\end{array}$ & $\begin{array}{c}\text { Number of } \\
\text { meas./night }\end{array}$ & $\begin{array}{c}\text { Exposure } \\
\text { time [min] }\end{array}$ & $\begin{array}{c}\text { Total time } \\
\text { per night [min] }\end{array}$ \\
\hline 2 & 3 & 10 & 30 \\
2 & 3 & 20 & 60 \\
5 & 2 & 15 & 30 \\
5 & 2 & 30 & 60 \\
24 & 1 & 15 & 15 \\
24 & 1 & 30 & 30 \\
\hline
\end{tabular}

\subsection{Setting new observational strategies}

In the previous section, we presented the result expected when using the current HARPS observational strategy for the highprecision subprogram (one measurement per night of $15 \mathrm{~min}$ ). The goal of our work is to determine whether we can reduce the noise yet further by sampling appropriately the timescales of the various intrinsic stellar noise sources. The following additional strategies have been tested: three measurements per night with $2 \mathrm{~h}$ of spacing and two measurements per night $5 \mathrm{~h}$ apart. For the first strategy, we tested exposure times of 10 and 20 min and for the second one, 15 and $30 \mathrm{~min}$. In addition, a strategy with one measurement per night of $30 \mathrm{~min}$ was tested. For longperiod planet investigations, we moreover need to follow the star as long as possible during the year to obtain a good coverage in phase. Because of airmass limitations, we also consider that stars can only be observed at most $5 \mathrm{~h}$ per night in good conditions. This explains the choice of the selected approaches. Table 4 presents the different strategies used in the simulations.

The strategy for 15-min total exposure times per night corresponds to that of the high-precision HARPS-GTO subprogram. The corresponding results are useful to compare with results obtained with the newly considered approaches. The different exposure times considered provide us with a good feeling of how we should average stellar oscillation effects ( $p$-modes that have a typical timescale of a few minutes for solar-type stars), whereas the multiple exposures during the night provide information about possible dumping, after binning, of granulation phenomena effects at lower frequencies.

\section{Results for the selected observational strategies}

We focus on the improvement brought about by the new strategies. First, we calculate the level of RV noise, $\mathrm{rms}_{\mathrm{RVb}}$, as a function of the binning in days. We use the same calendar as in the previous section, which will allow us to compare the new strategies with the present one used on HARPS.

\subsection{An efficient and affordable observational strategy}

The evolution of the RV variation, $\mathrm{rms}_{\mathrm{RVb}}$, as a function of the binning in days is shown in Fig. 4. In these plots, each type of line corresponds to the same number of measurements per night. For each of them, we have 2 curves, the lower one is obtained with an exposure time twice as long as the upper one. The difference between these 2 curves is small, so doubling the exposure time does not strongly improve the results, although it doubles the total measurement cost (neglecting overheads). Thus, it appears that the exposure time is not the only parameter that can average out stellar noise. The frequency of measurements also plays an important role. 

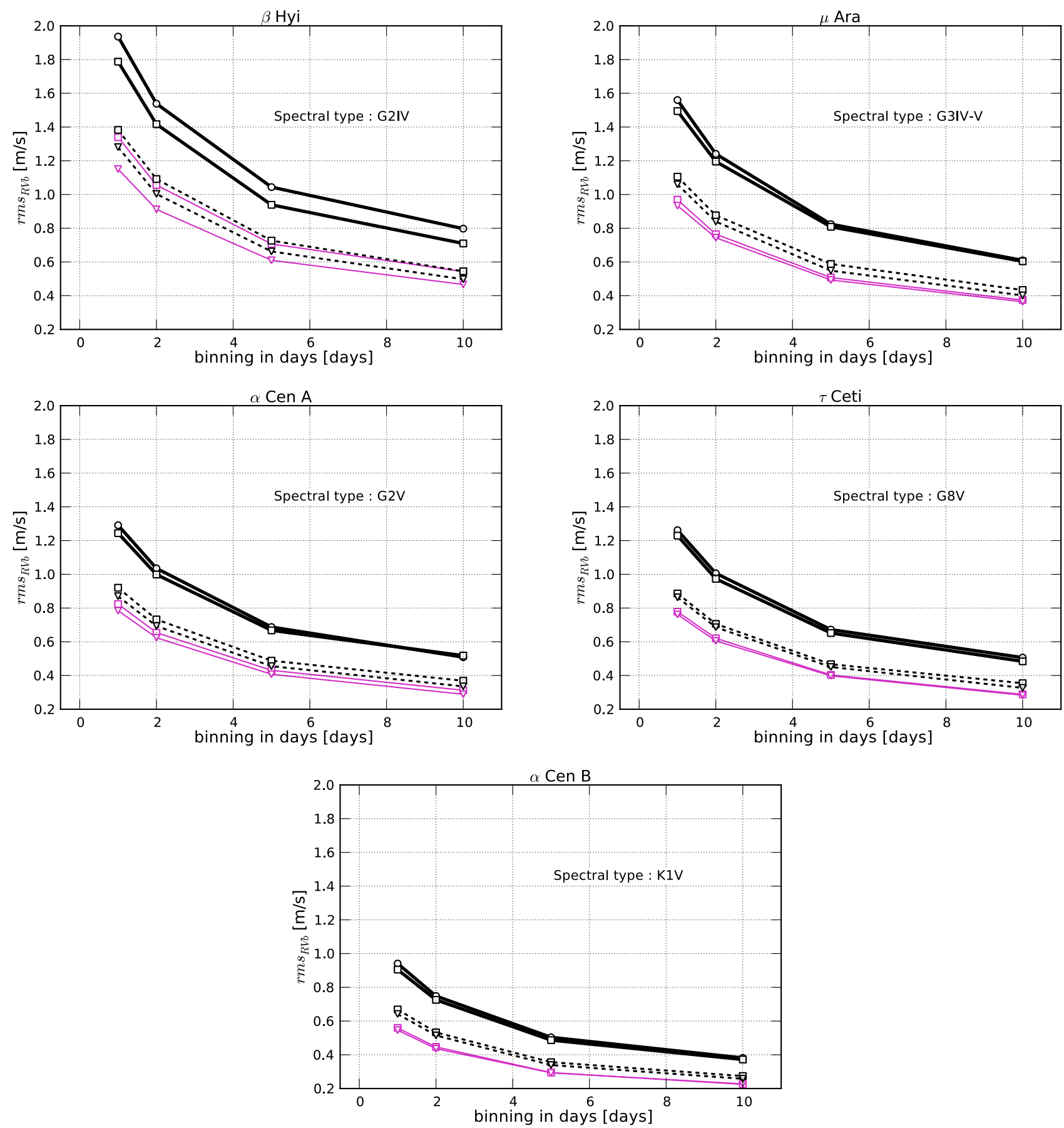

Fig. 4. Rms of the binned RVs, $\mathrm{rms}_{\mathrm{RVb}}$, as a function of the binning in days. The different considered strategies are one measurement per night (the 2 heavy continuous lines), 2 measurements per night with a spacing of $5 \mathrm{~h}$ (the 2 dashed lines) and 3 measurements per night with a spacing of $2 \mathrm{~h}$ (the 2 thin continuous lines). Circles represent a total observing time of $15 \mathrm{~min}$ per night $(1 \times 15 \mathrm{~min})$, squares represent a total observing time of $30 \mathrm{~min}$ per night $(1 \times 30 \mathrm{~min}, 2 \times 15 \mathrm{~min}$, and $3 \times 10 \mathrm{~min})$ and triangles represent a total observing time of $1 \mathrm{~h}$ per night $(2 \times 30 \mathrm{~min}$ and $3 \times 20 \mathrm{~min})$.

Taking only the shortest exposure times, we are left with three strategies: three times 10-min measurements per night with $2 \mathrm{~h}$ of spacing between them (hereafter, 3N strategy), two times 15-min measurements per night separated by $5 \mathrm{~h}$ (hereafter, 2N strategy), and one measurement per night with a 15 min exposure time (hereafter, $1 \mathrm{~N}$ strategy). Comparing the one measurement-per-night of the 30 min strategy with the $2 \mathrm{~N}$ and $3 \mathrm{~N}$ strategies allows us to clearly see that with a similar observation time per night (30 $\mathrm{min})$, separating the measurements over the night into 2 or 3 blocks significantly improves the averaging of the stellar noise. The most effective of the considered strategies is the $3 \mathrm{~N}$ strategy. This strategy gives values for $\mathrm{rms}_{\mathrm{RVb}}$ that are on average 30 to $40 \%$ smaller than the present high precision strategy used on HARPS.

As already pointed before, the RV noise due to stellar intrinsic perturbations is lower for $\mathrm{K}$ than $\mathrm{G}$ dwarfs. Within a given spectral type, we can also compare this value between a main sequence and a more evolved star ( $\alpha$ Cen A (G2V) and $\mu$ Ara (G3IV-V) or $\beta$ Hyi (G2IV)). For more evolved stars, the RV variation seems higher, though we need to be careful because we 
X. Dumusque et al.: Stellar noise and planetary detection. I.

Table 5. Spectral type, activity level, and rms of the residuals, $\mathrm{Rms}_{\mathrm{O}-\mathrm{C}}$, for known planetary systems discovered using HARPS.

\begin{tabular}{ccccc}
\hline \hline Star & Spectral type & $\log \left(R_{\mathrm{HK}}^{\prime}\right)$ & $\mathrm{Rms}_{\mathrm{O}-\mathrm{C}}\left[\mathrm{ms}^{-1}\right]$ & Reference \\
\hline HD 69830 & K0V & -4.97 & 0.81 & Lovis et al. (2006) \\
HD 40307 & K2.5V & -4.99 & 0.85 & Mayor et al. (2009b) \\
HD 47186 & G5V & -5.01 & 0.91 & Bouchy et al. (2009) \\
HD 4308 & G5V & -4.93 & 1.3 & Udry et al. (2006) \\
HD 181720 & G1V & -5.01 & 1.37 & Santos et al. (2010) \\
\hline
\end{tabular}

have only one target, $\beta$ Hyi ( $\mu$ Ara is between a dwarf and a subgiant).

\subsection{Strategy effects for the different stellar noise sources}

The impact of exposure time and frequency of measurements on each kind of intrinsic stellar noise can be obtained by separately considering each noise contribution to the power spectrum. Since we fit each type of noise, we can extract independently the 3 types of granulation phenomena noises and the noise coming from oscillation modes. Using the technique to obtain synthetic RV measurements, we can then create data containing each type of noise, apply different strategies to them, and thus see the impact of varying the exposure time and the frequency of measurements on each kind of stellar noise.

Unsurprisingly, p-modes, causing oscillations of the solar surface over timescales shorter than 15 min can be adequately averaged by longer exposure times ${ }^{7}$. For noise of slightly lower frequency, it is useful to increase the total exposure time as much as possible $(\sim 30 \mathrm{~min})$. This will average the effect of granulation whose typical timescale is shorter than $\sim 25 \mathrm{~min}$. For the mesogranulation, a further increase in the exposure time would be ineffective whereas observing the star more often during the night averages out more efficiently the stellar noise after binning. The $3 \mathrm{~N}$ strategy is the one providing the best results for mesogranulation, since the $2 \mathrm{~h}$ of spacing between the measurements over the night appropriately samples the perturbation timescale of the phenomenon. Finally, for supergranulation at even longer timescales, taking two measurements with a spacing of $5 \mathrm{~h}$ averages out most efficiently this long-term stellar noise.

\section{Comparison with real observations}

Our simulation only takes into account oscillations, granulation phenomena, and both instrumental and photon noises. Lower frequency variations, originating in active regions, should produce a higher level of noise, even for stars in their minimum activity state (Meunier et al. 2010). To check whether our simulation, disregarding active regions, corresponds to a realistic case, we have to compare the level of noise simulated with long-term jitter observed on other stars.

Our simulation provides the noise expected for a given observational strategy. To compare this simulated noise with real observations, we have to use an observational strategy similar to the one nowadays used on HARPS. We thus choose the $1 \mathrm{~N}$ strategy. Using this strategy for both $\alpha$ Cen A and $\alpha$ Cen B with no binning, the dispersion taking into account stellar, instrumental, and photon noise is equal to 1.29 and $0.94 \mathrm{~m} \mathrm{~s}^{-1}$, respectively. These values can be compared to the rms of the residuals obtained for

\footnotetext{
7 Regardless of whether we take one measurement per night of $15 \mathrm{~min}$ or 3 measurements per night of $5 \mathrm{~min}$ and perform some binning, our results are similar.
}

detected planetary systems. Table 5 gives the rms of the residuals for host stars of spectral type similar to $\alpha$ Cen A (G2V) and $\alpha$ Cen B (K1V). The obtained values are in good agreement with observations, which indicate that our simulation of stellar and instrumental noises are realistic and that stars with noise dominated by high and moderate frequency variations exist.

\section{Detection limits in the mass-period diagram}

To derive detection limits in terms of planet mass and period accessible with the different studied strategies, we calculate the false alarm probability (FAP) of detection using bootstrap randomization (Endl et al. 2001; Efron \& Tibshirani 1998). We first simulate a synthetic RV set containing stellar, instrumental, and photon noise as we did in the previous sections. The calendar used (see Sect. 4.1) is the same, with 256 nights over a time span of 4 years. We then carry out 1000 bootstrap randomizations ${ }^{8}$ of these RVs and calculate the corresponding periodograms. For each periodogram, we select the highest peak and construct a distribution of these 1000 highest peaks. The $1 \%$ FAP corresponds to the power that is only reached $1 \%$ of the time. The second step consists of adding a sinusoidal signal with a given period to the synthetic RVs. We calculate the periodogram of these new RVs and compare the height of the observed peak with the 1\% FAP for the given period. We then adjust the semiamplitude of the signal until the power of the peak is equal to the $1 \%$ FAP. The semi-amplitude obtained corresponds to the detection limit of a null eccentricity planet with a confidence level of $99 \%$. To be conservative, we test 10 different phases and select the highest semi-amplitude value.

In practice, we simulate $100 \mathrm{RV}$ sets for each strategy, and only the 10 "worst" cases were considered in the end. In Fig. 5, we can see the mass detection limits, with a confidence level of $99 \%$, for the $1 \mathrm{~N}, 2 \mathrm{~N}$, and $3 \mathrm{~N}$ strategies using HARPS. In each graph, we can discern a peak of the mass detection limits in one year. This could be explained by the 4 months removed each year in the calendar to take into account that stars disappear from the sky. Signals of a one year period are not well-sampled, which leads to a local increase in the detection limit.

Among the three strategies studied, we note that the use of the $2 \mathrm{~N}$ and $3 \mathrm{~N}$ strategies allows us to decrease the level of detection limits by approximatively $2 M_{\oplus}$, compared to the high precision strategy normally used on HARPS (1N strategy). This improvement is not negligible, since it reduces mass detection limits by approximately $30 \%$. The difference between the $2 \mathrm{~N}$ and the $3 \mathrm{~N}$ strategy is small, but as shown before in Sect. 5.1, the $3 \mathrm{~N}$ strategy seems to be the most efficient one in averaging out the considered stellar noise.

In Fig. 5, stars are ordered from top to bottom and left to right from early spectral type and evolved to late spectral type and

8 Following the use of this well-sampled calendar, we choose to calculate the FAP using bootstrap randomization rather than permutation since it is more efficient in this case (Efron \& Tibshirani 1998). 

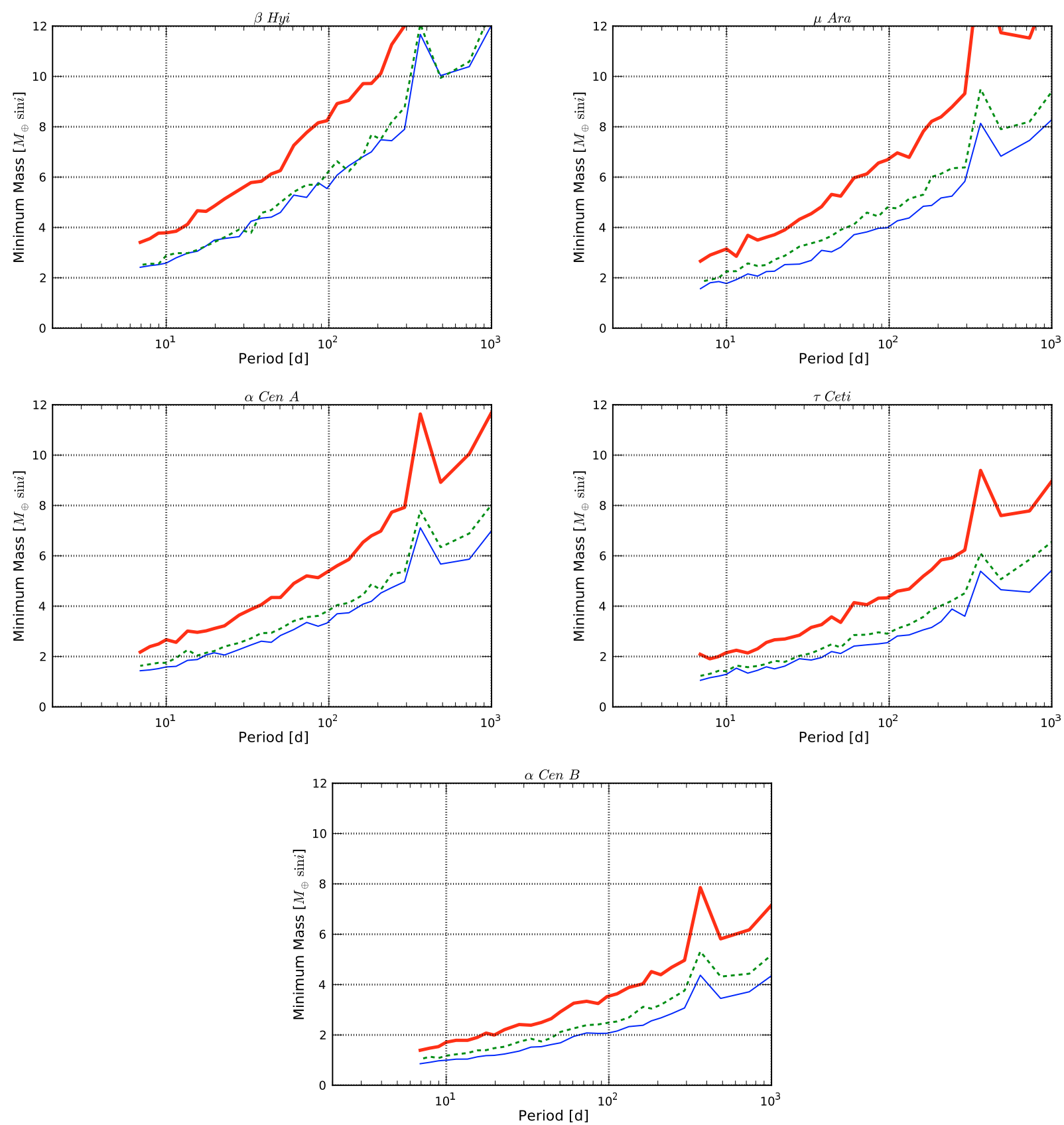

Fig. 5. Mass-period diagrams for our star sample. We can see on each graph, the 3 strategies studied. one measurement of 15 min per night (continuous thick line), 2 measurements per night of $15 \mathrm{~min} 5 \mathrm{~h}$ apart (dashed line) and 3 measurements per night of $10 \mathrm{~min} 2 \mathrm{~h}$ apart (continuous thin line).

not evolved. Following this order, we note that the level of mass detection limits decreases. Thus, $\alpha$ Cen B, which is a $\mathrm{K} 1 \mathrm{dwarf}$ star, has the lowest mass detection limits in our sample.

If we only consider the dwarf stars, $\alpha$ Cen A (G2V), $\tau$ Ceti (G8V), and $\alpha$ Cen B (K1V), we noce that the level of the mass detection limits decreases when we go towards late spectral types. For example, for a 100 day period, the mass detection limit for the $3 \mathrm{~N}$ strategy will be $3.5 M_{\oplus}, 2.5 M_{\oplus}$, and $2 M_{\oplus}$ for the $\mathrm{G} 2 \mathrm{~V}$, the $\mathrm{G} 8 \mathrm{~V}$, and the $\mathrm{K} 1 \mathrm{~V}$ star, respectively. Thus, late spectral type stars have lower mass detection limits, which can be explained by two phenomena. The first obvious one is that early $\mathrm{K}$ dwarfs will be less massive than $\mathrm{G}$ dwarfs. Therefore, for a given mass planet, the RV signal will have a higher variation for a late spectral type star. Nevertheless, this effect is not sufficient to explain this large range of mass detection limits ${ }^{9}$ and the second phenomena, which is the most important one, is that early $\mathrm{K}$ dwarfs have a lower level of stellar noise than $\mathrm{G}$ dwarfs (see Table 3 and Fig. 4).

If we now look, in the same spectral-type range, at stars with different evolutionary states, we note an interesting behavior. $\beta$ Hyi is clearly a G2 subgiant, $\mu$ Ara is a G3 star in the transition between the dwarf and the subgiant branch (Soriano \& Vauclair 2010), whereas $\alpha$ Cen A is a G2 dwarf. Studying these 3 different cases, we note that the level of mass detection limit is higher for evolved stars. For example, the subgiant $\beta$ Hyi has mass detection limits 1.5 times higher than the dwarf $\alpha$ Cen A.

\footnotetext{
${ }_{9} \alpha$ Cen B as a mass of $0.90 M_{\odot}$, which is slightly smaller than $\alpha$ Cen A, $1.09 M_{\odot}$
} 
Following these results for dwarf and sub-giant stars, it seems clear that to find the lowest mass planets using RVs, we should search around early K dwarfs. Observation of these stars are affected by the lowest level of stellar noise, thus the lowest mass detection limits. Besides this, early $\mathrm{K}$ dwarfs are also interesting because their habitable zones, around 200 days, are closer than for early $\mathrm{G}$ dwarfs. For stars such as $\alpha$ Cen B (K1V) (see Fig. 5), the simulations predict that we should be able to find planets of $2.5 M_{\oplus}$ in the habitable zone, using the $3 \mathrm{~N}$ strategy.

As already pointed out, because of a short time span of the asteroseismology measurements, stellar activity noise is not fully included in our simulation. Thus, the present limits indicate the RV signal that can be reliably detected when activity noise is negligible. In the presence of real stellar activity noise, the detection limits will increase. This will be the topic in a forthcoming paper.

\section{Detection limits using a real HARPS observational calendar}

To check whether the calendar we use is not too idealistic compared to a real one (see Sect. 4.1), we compute the detection limits using the real calendar of one of the most well-studied star using HARPS, HD 69830. This real calendar regroups a total of 157 nights over an observing span of 1615 days (Lovis et al. 2006, 2010, in prep.). Figure 6 shows the difference in mass detection limits between the calendar used in this paper and the real one for HD 69830. For a 100 day period, the detection limit of the two calendars are 2 and $2.5 M_{\oplus}$, respectively. This small increase of $25 \%$ is due only to the total number of nights present in each calendar. The semi-amplitude in RV is proportional to the square root of the total number of measurements. Therefore, changing from 256 nights to 157 will increase the detection limit by $28 \%$, very close to that inferred by the simulation. Applying the $3 \mathrm{~N}$ strategy to a K dwarf, such as HD 69830, should lead to the detection of $3 M_{\oplus}$ planets in their habitable zone. Thus, by using a real HARPS observational calendar, we can reach similar detection limits. A real observational calendar depends not only on bad weather and instrumental problems, but also on the time allocated for each star. The present strategy for HARPS is to follow many targets, which reduces the number of measurements per star. In searching for the lowest mass planets, only a very small sample of stars should be selected. This would allow us to increase the number of measurements from those taken in a real observational HARPS calendar. This is why the simulations performed in the previous section uses 256 nights of measurements over 4 years rather than 157 .

\section{Concluding remarks}

The present high-precision HARPS observational strategy (one measurement of $15 \mathrm{~min}$ per night, $1 \mathrm{~N}$ strategy in the paper) suceeds in reducing the stellar oscillation noise, but is not optimized to average out granulation phenomena (granulation, mesogranulation, and supergranulation). These latter sources of noise perturb radial velocity measurements on timescales of up to 1.5 days. Increasing the number of measurements per night allows us to reduce the effect of granulation phenomena, after binning, and consequently improve the planetary detection limits. The observational strategy that averages out most efficiently the considered stellar noise, three measurements per night each $2 \mathrm{~h}$ with an exposure time of $10 \mathrm{~min}$ each $(3 \mathrm{~N}$ strategy in the paper), gives detection limits on average that are $30 \%$ better than

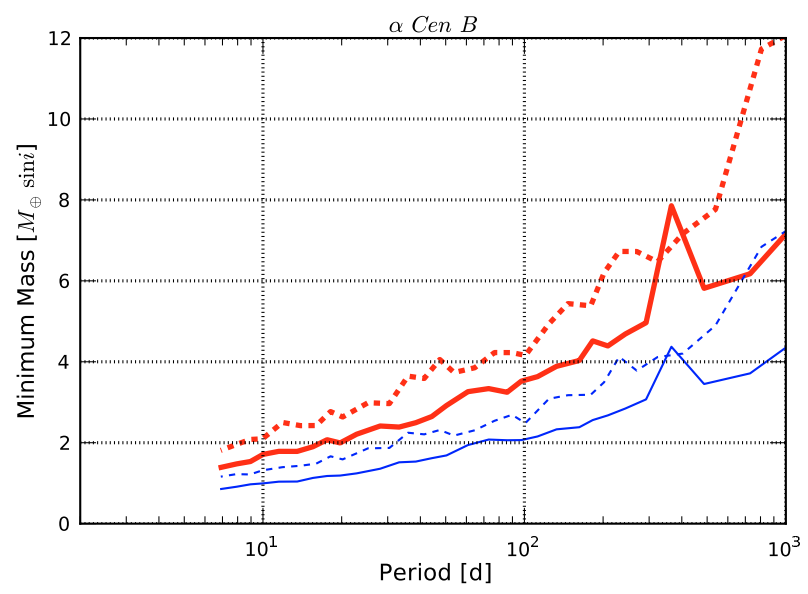

Fig. 6. Mass-period diagrams for $\alpha$ Cen B. Thick and thin lines represent the $1 \mathrm{~N}$ and the $3 \mathrm{~N}$ strategy, respectively. Plain lines correspond to the simulated calendar and dashed lines to the real calendar of HD 69830.

the present HARPS high-resolution strategy. This improvement is really due to choosing to make the three measurements per night and not to the total observational time (which is twice that of the present HARPS observational strategy). We have shown that one measurement per night of $30 \mathrm{~min}$ is inefficient.

Our work also suggests a trend between the level of the stellar noise considered in this study and the spectral type of dwarf stars. Early K dwarfs have a lower total noise level than early $\mathrm{G}$ dwarfs. We have also identified a trend between evolved and non-evolved stars, non-evolved stars having a lower level of stellar noise. Therefore, early $\mathrm{K}$ dwarfs appears to have the lowest level of stellar noise of our sample and consequently the lowest planet-mass detection limits. In addition, the habitable zone is closest in early $\mathrm{K}$ than in early $\mathrm{G}$ dwarfs, making early $\mathrm{K}$ dwarfs the most promising targets to search for very low mass planets.

Following the conclusion of the last two paragraphs, we applied the three measurements per night strategy to $\alpha$ Cen B $(\mathrm{K} 1 \mathrm{~V})$. For the habitable zone of this planet corresponding to approximately 200 days, our simulation shows that we would be able to detect planets of $3 M_{\oplus}$ using an existing HARPS observational calendar. Therefore, granulation phenomena and oscillation modes will not prevent us from finding Earth-like planets in habitable regions.

However, there are two different limitations to our present study. First of all, owing to the short time-span measurements, we do not properly take into account stellar activity noise sources, which can be important. The detection limits we obtain are only valid for stars without significant activity-related phenomena, such as spots and plages. We have shown that these stars exist and therefore activity will not be a problem for some targets. A second limitation may be that we only simulate the presence of one planet per star. The majority of small planets discovered nowadays are in multiple systems, which complicates the analysis. More data is required in these cases to allow us to fit all the free parameters.

Next generation spectrographs, such as ESPRESSO (http://espresso.astro.up.pt/) and CODEX (e.g. Pasquini et al. 2008) will reach higher levels of precision and stability, most likely leading to a reduction in detection limits near $1 M_{\oplus}$.

Acknowledgements. We would like to thank Tim Bedding for providing us the power spectrum and the window function of $\beta$ Hyi. N.C. Santos would like to thank the support by the European Research Council/European 
Community under the FP7 through a Starting Grant, as well as the support from Fundação para a Ciência e a Tecnologia (FCT), Portugal, through programme Ciência 2007. We would also like to acknowledge support from FCT in the form of grants reference PTDC/CTE-AST/098528/2008 and PTDC/CTEAST/098604/2008. Finally, this work was supported by the European Helioand Asteroseismology Network (HELAS), a major international collaboration funded by the European Commission's Sixth Framework Program (grant: FP62004-Infrastructures-5-026183).

\section{References}

Andersen, B. N., Leifsen, T. E., \& Toutain, T. 1994, Sol. Phys., 152, 247 Arentoft, T., Kjeldsen, H., Bedding, T. R., et al. 2008, ApJ, 687, 1180 Bazot, M., Vauclair, S., Bouchy, F., \& Santos, N. C. 2005, A\&A, 440, 615 Bazot, M., Bouchy, F., Kjeldsen, H., et al. 2007, A\&A, 470, 295

Bedding, T. R., Kjeldsen, H., Arentoft, T., et al. 2007, ApJ, 663, 1315

Bouchy, F., Bazot, M., Santos, N. C., Vauclair, S., \& Sosnowska, D. 2005, A\&A, 440, 609

Bouchy, F., Mayor, M., Lovis, C., et al. 2009, A\&A, 496, 527

Brandt, P. N., \& Solanki, S. K. 1990, A\&A, 231, 221

Broomhall, A., Chaplin, W. J., Davies, G. R., et al. 2009, MNRAS, 396, L100

Butler, R. P., Bedding, T. R., Kjeldsen, H., et al. 2004, ApJ, 600, L75

Carrier, F., \& Eggenberger, P. 2006, A\&A, 450, 695

Chapman, G. A., Cookson, A. M., Dobias, J. J., \& Walton, S. R. 2001, ApJ, 555, 462

Christensen-Dalsgaard, J. 2004, Sol. Phys., 220, 137

Del Moro, D. 2004, A\&A, 428, 1007

Del Moro, D., Berrilli, F., Duvall, Jr., T. L., \& Kosovichev, A. G. 2004, Sol. Phys., 221, 23

Dravins, D. 1982, ARA\&A, 20, 61

Efron, B., \& Tibshirani, R. J. 1998, An Introduction to the Bootstrap (Chapman \& Hall/CRC)

Endl, M., Kürster, M., Els, S., Hatzes, A. P., \& Cochran, W. D. 2001, A\&A, 374, 675

Gray, D. F. 1992, The Observation and Analysis of Stellar Photospheres, ed. D. F. Gray, June (Cambridge, UK: Cambridge University Press), 470
Harvey, J. W. 1984, in probing the depths of star: the study of Solar oscillation from space, ed. R. W. Noyes, \& E. J. Rhodes Jr., Pasadena, JPL/NASA, 327

Huélamo, N., Figueira, P., Bonfils, X., et al. 2008, A\&A, 489, L9

Kjeldsen, H., \& Bedding, T. R. 1995, A\&A, 293, 87

Kjeldsen, H., Bedding, T. R., Butler, R. P., et al. 2005, ApJ, 635, 1281

Kjeldsen, H., Bedding, T. R., Arentoft, T., et al. 2008, ApJ, 682, 1370

Lagrange, A., Desort, M., \& Meunier, N. 2010, A\&A, 512, A38

Lefebvre, S., García, R. A., Jiménez-Reyes, S. J., Turck-Chièze, S., \& Mathur, S. 2008, A\&A, 490, 1143

Livingston, W. C. 1982, Nature, 297, 208

Lomb, N. R. 1976, Ap\&SS, 39, 447

Lovis, C., Mayor, M., Pepe, F., et al. 2006, Nature, 441, 305

Mayor, M., Pepe, F., Queloz, D., et al. 2003, The Messenger, 114, 20

Mayor, M., Bonfils, X., Forveille, T., et al. 2009a, A\&A, 507, 487

Mayor, M., Udry, S., Lovis, C., et al. 2009b, A\&A, 493, 639

Meunier, N., Desort, M., \& Lagrange, A. 2010, A\&A, 512, A39

O’Toole, S. J., Tinney, C. G., \& Jones, H. R. A. 2008, MNRAS, 386, 516

Palle, P. L., Jimenez, A., Perez Hernandez, F., et al. 1995, ApJ, 441, 952

Pasquini, L., Avila, G., Dekker, H., et al. 2008, in Society of Photo-Optical Instrumentation Engineers (SPIE) Conf. Ser., 7014

Pepe, F., Mayor, M., Queloz, D., et al. 2005, The Messenger, 120, 22

Pepe, F., Correia, A. C. M., Mayor, M., et al. 2006, A\&A, 462, 769

Queloz, D., Henry, G. W., Sivan, J. P., et al. 2001, A\&A, 379, 279

Saar, S. H., \& Donahue, R. A. 1997, ApJ, 485, 319

Santos, N. C., Bouchy, F., Mayor, M., et al. 2004, A\&A, 426, L19

Santos, N. C., Mayor, M., Benz, W., et al. 2010, A\&A, 512, A47

Scargle, J. D. 1982, ApJ, 263, 835

Schrijver, C. J., \& Zwann, C. 2000, Solar and stellar magnetic activity (Cambridge U Press)

Soriano, M., \& Vauclair, S. 2010, A\&A, 513, A49

Sousa, S. G., Santos, N. C., Mayor, M., et al. 2008, A\&A, 487, 373

Tassoul, M. 1980, ApJS, 43, 469

Teixeira, T. C., Kjeldsen, H., Bedding, T. R., et al. 2009, A\&A, 494, 237

Title, A. M., Tarbell, T. D., Topka, K. P., et al. 1989, ApJ, 336, 475

Udry, S., Mayor, M., Benz, W., et al. 2006, A\&A, 447, 361

Udry, S., Bonfils, X., Delfosse, X., et al. 2007, A\&A, 469, L43

Zechmeister, M., \& Kürster, M. 2009, A\&A, 496, 577 